\title{
Multiple Positive Solutions for \\ a Quasilinear Elliptic System Involving Concave-Convex Nonlinearities and Sign-Changing Weight Functions
}

\author{
Tsing-San Hsu \\ Center for General Education, Chang Gung University, Kwei-Shan, Tao-Yuan 333, Taiwan
}

Correspondence should be addressed to Tsing-San Hsu, tshsu@mail.cgu.edu.tw

Received 20 April 2011; Accepted 20 December 2011

Academic Editor: Marco Squassina

Copyright (c) 2012 Tsing-San Hsu. This is an open access article distributed under the Creative Commons Attribution License, which permits unrestricted use, distribution, and reproduction in any medium, provided the original work is properly cited.

Let $\Omega \ni 0$ be an-open bounded domain in $\mathbb{R}^{N}(N \geq 3)$ and $p^{*}=(p N /(N-p))$. We consider the following quasilinear elliptic system of two equations in $W_{0}^{1, p}(\Omega) \times W_{0}^{1, p}(\Omega):-\Delta_{p} u=\lambda f(x)|u|^{q-2} u+$ $(\alpha /(\alpha+\beta)) h(x)|u|^{\alpha-2} u|v|^{\beta},-\Delta_{p} v=\mu g(x)|v|^{q-2} v+(\beta /(\alpha+\beta)) h(x)|u|^{\alpha}|v|^{\beta-2} v$, where $\lambda, \mu>0, \Delta_{p}$ denotes the $p$-Laplacian operator, $1 \leq q<p<N, \alpha, \beta>1$ satisfy $p<\alpha+\beta \leq p^{*}$, and $f, g, h$ are continuous functions on $\bar{\Omega}$ which are somewhere positive but which may change sign on $\Omega$. We establish the existence and multiplicity results of positive solutions to (the above mentioned quasilinear elliptic system equations) by variational methods.

\section{Introduction and Main Results}

Let $\Omega \ni 0$ be a smooth-bounded domain in $\mathbb{R}^{N}$ with $N \geq 3$. In this paper, we study the existence and multiplicity of positive solutions for the following quasilinear elliptic system:

$$
\begin{gathered}
-\Delta_{p} u=\lambda f(x)|u|^{q-2} u+\frac{\alpha}{\alpha+\beta} h(x)|u|^{\alpha-2} u|v|^{\beta} \quad \text { in } \Omega, \\
-\Delta_{p} v=\mu g(x)|v|^{q-2} v+\frac{\beta}{\alpha+\beta} h(x)|u|^{\alpha}|v|^{\beta-2} v \quad \text { in } \Omega, \quad\left(S_{\lambda f, \mu g, h}\right) \\
u=v=0 \quad \text { on } \partial \Omega,
\end{gathered}
$$


where $\lambda, \mu>0,1 \leq q<p<N, \Delta_{p} u=\operatorname{div}\left(|\nabla u|^{p-2} \nabla u\right)$ is the $p$-Laplacian, $\alpha>1, \beta>1$ satisfy $p<\alpha+\beta \leq p^{*}, p^{*}=(p N) /(N-p)$ denotes the critical Sobolev exponent, and the weight functions $f, g, h$ are satisfying the following assumptions:

(A1) $f, g \in C(\bar{\Omega}), f^{+}=\max \{f, 0\} \not \equiv 0, g^{+}=\max \{g, 0\} \not \equiv 0$ and $|f|_{\infty}=|g|_{\infty}=1$;

(A2) $h \in C(\bar{\Omega}), h^{+}=\max \{h, 0\} \not \equiv 0$ and $|h|_{\infty}=1$;

(A3) there exist $a_{0}, b_{0}, r_{0}>0$ and $x_{0} \in \Omega$ such that $B\left(x_{0}, 2 r_{0}\right) \subset \Omega, f(x) \geq a_{0}$ and $g(x) \geq b_{0}$ for all $x \in B\left(x_{0}, 2 r_{0}\right)$, without loss of generality, we assume below that $x_{0}=0$;

(A4) $h(x)>0$ for all $x \in \Omega,|h|_{\infty}=h(0)$ and there exists $\delta_{0}>N /(p-1)$ such that

$$
h(x)=h(0)+o\left(|x|^{\delta_{0}}\right) \quad \text { as } x \longrightarrow 0 .
$$

System $\left(S_{\lambda f, \mu g, h}\right)$ is posed in the framework of the Sobolev space $W=W_{0}^{1, p}(\Omega) \times W_{0}^{1, p}(\Omega)$ with the standard norm

$$
\|(u, v)\|=\left(\int_{\Omega}|\nabla u|^{p} d x+\int_{\Omega}|\nabla v|^{p} d x\right)^{1 / p}
$$

Moreover, a pair of functions $(u, v) \in W$ is said to be a weak solution of System $\left(S_{\lambda f, \mu g, h}\right)$ if

$$
\begin{aligned}
\int_{\Omega}|\nabla u|^{p-2} \nabla u \nabla \varphi_{1} d x+\int_{\Omega}|\nabla v|^{p-2} \nabla v \nabla \varphi_{2} d x \\
\quad-\lambda \int_{\Omega} f(x)|u|^{q-2} u \varphi_{1} d x-\mu \int_{\Omega} g(x)|v|^{q-2} v \varphi_{2} d x \\
\quad-\frac{\alpha}{\alpha+\beta} \int_{\Omega} h(x)|u|^{\alpha-2} u|v|^{\beta} \varphi_{1} d x-\frac{\beta}{\alpha+\beta} \int_{\Omega} h(x)|u|^{\alpha}|v|^{\beta-2} v \varphi_{2} d x=0
\end{aligned}
$$

for all $\left(\varphi_{1}, \varphi_{2}\right) \in W$. Thus, the corresponding energy functional of System $\left(S_{\lambda f, \mu g, h}\right)$ is defined by

$$
I_{\lambda, \mu}(u, v)=\frac{1}{p}\|(u, v)\|^{p}-\frac{1}{q} \int_{\Omega}\left(\lambda f(x)|u|^{q}+\mu g(x)|v|^{q}\right) d x-\frac{1}{\alpha+\beta} \int_{\Omega} h(x)|u|^{\alpha}|v|^{\beta} d x .
$$

Semilinear and quasilinear scalar elliptic equations with concave-convex nonlinearities are widely studied: we refer the reader to Ambrosetti et al. [1], de Figueiredo et al. [2], Azorero and Peral [3], Azorero et al. [4], EL Hamidi [5], Hirano et al. [6], Hsu [7], and Wu [8], and so forth. For the nonlinear elliptic systems, we refer to Adriouch and EL Hamidi [9], Ahammou [10], Alves et al. [11], Bozhkov and Mitidieri [12], Clément et al. [13], de Figueiredo and Felmer [14], EL Hamidi [15], Hsu and Lin [16, 17], Squassina [18], Vélin [19], and $\mathrm{Wu}[20]$, and so forth.

Recently, in [8], the author has considered a semilinear scalar elliptic equation involving concave-convex nonlinearities and sign-changing weight functions and showed multiplicity results with respect to the parameter via the extraction of Palais-Smale sequences 
in the Nehair manifold, and for the definition of Nehair manifold we refer the reader to see Nehari [21] or Willem [22].

More recently, in [20] the author extends the method of [8] to system $\left(S_{\lambda f, \mu g, h}\right)$ in the semilinear case $p=2$ with the subcritical case $2<\alpha+\beta<2^{*}$ and the sign-changing weight functions $f, g$. In [16] the author also extends the method of [8] to system $\left(S_{\lambda f, \mu g, h}\right)$ in the quasilinear case $1<p<N$ with critical case $\alpha+\beta=p^{*}$ and the constant weight functions $f \equiv g \equiv h \equiv 1$. In the present paper, motivated by $[16,20]$ we extend and improve the papers by $\mathrm{Hsu}[16]$ and $\mathrm{Wu}[20]$. First, we deal with more general weight functions $f, g, h$ which may be changing sign, and second, we also deal with quasilinear elliptic systems involving subcritical or critical Sobolev exponents. by

Let $S$ be the best Sobolev constant for the embedding of $W_{0}^{1, p}(\Omega)$ in $L^{\alpha+\beta}(\Omega)$ defined

$$
S=\inf _{u \in W_{0}^{1, p}(\Omega) \backslash\{0\}} \frac{\int_{\Omega}|\nabla u|^{p} d x}{\left(\int_{\Omega}|u|^{\alpha+\beta} d x\right)^{p /(\alpha+\beta)}}
$$

and set

$$
\Lambda_{1}=\left(\frac{p-q}{\alpha+\beta-q}\right)^{p /(\alpha+\beta-p)}\left(\frac{\alpha+\beta-q}{\alpha+\beta-p}|\Omega|^{(\alpha+\beta-q) /(\alpha+\beta)}\right)^{-p /(p-q)} S^{p(\alpha+\beta-q) /(p-q)(\alpha+\beta-p)}>0,
$$

where $|\Omega|$ is the Lebesgue measure of $\Omega$. Our main results are as follows.

Theorem 1.1 (existence of one positive solution). Assume that (A1)-(A2) hold. If $1 \leq q<p<$ $N, p<\alpha+\beta \leq p^{*}$, and $\lambda, \mu>0$ satisfy $0<\lambda^{p /(p-q)}+\mu^{p /(p-q)}<\Lambda_{1}$, then system $\left(S_{\lambda f, \mu g, h}\right)$ has at least one positive solution in $W_{0}^{1, p}(\Omega) \times W_{0}^{1, p}(\Omega)$.

Theorem 1.2 (second positive solution in the subcritical case). Assume that (A1)-(A2) hold. If $1 \leq q<p<N, p<\alpha+\beta<p^{*}$, and $\lambda, \mu>0$ satisfy $0<\lambda^{p /(p-q)}+\mu^{p /(p-q)}<(q / p)^{p /(p-q)} \Lambda_{1}$, then System $\left(S_{\lambda f, \mu g, h}\right)$ has at least two positive solutions in $W_{0}^{1, p}(\Omega) \times W_{0}^{1, p}(\Omega)$.

Theorem 1.3 (second positive solution in the critical case). Assume that (A1)-(A4) hold. If $1 \leq q<p<N, \alpha+\beta=p^{*}$, and $\lambda, \mu>0$ satisfy $0<\lambda^{p /(p-q)}+\mu^{p /(p-q)}<(q / p)^{p /(p-q)} \Lambda_{1}$, then System $\left(S_{\lambda f, \mu g, h}\right)$ has at least two positive solutions in $W_{0}^{1, p}(\Omega) \times W_{0}^{1, p}(\Omega)$.

This paper is organized as follows. In Section 2, we give some notations and preliminaries. The proofs of Theorems 1.1 and 1.2 are in Section 3. In Section 4, we manage to give the proof of Theorem 1.3. Throughout this paper, $(A 1)$ and $(A 2)$ will be assumed.

\section{Notations and Preliminaries}

In this section, we give some notations and necessary preliminary results.

Notations. We make use of the following notation.

$L^{s}(\Omega), 1 \leq s<\infty$, denote Lebesgue spaces; the norm $L^{s}$ is denoted by $|\cdot|_{s}$ for $1 \leq s \leq \infty$; 
$W=\left[W_{0}^{1, p}(\Omega)\right]^{2}$, endowed with norm $\|z\|^{p}=\|(u, v)\|^{p}=|\nabla u|_{p}^{p}+|\nabla v|_{p}^{p} ;$

The dual space of a Banach space $W$ will be denoted by $W^{-1}$;

$t z=t(u, v)=(t u, t v)$ for all $z=(u, v) \in W$ and $t \in \mathbb{R}$;

$|z|=(|u|,|v|)$ for all $z=(u, v) \in W$;

$z=(u, v)$ is said to be nonnegative in $\Omega$ if $u \geq 0$ and $v \geq 0$ in $\Omega$;

$z=(u, v)$ is said to be positive in $\Omega$ if $u>0$ and $v>0$ in $\Omega$;

$|\Omega|$ is the Lebesgue measure of $\Omega$;

$B\left(x_{0}, r\right)=\left\{x \in \mathbb{R}^{N}|| x-x_{0} \mid<r\right\}$ is the ball in $\mathbb{R}^{N}$;

$O\left(\varepsilon^{t}\right)$ denotes $\left|O\left(\varepsilon^{t}\right)\right| / \varepsilon^{t} \leq C$ as $\varepsilon \rightarrow 0$ for $t \geq 0$;

$o_{n}(1)$ denotes $o_{n}(1) \rightarrow 0$ as $n \rightarrow \infty$;

$C, C_{i}$ will denote various positive constants, the exact values of which are not important;

$p^{*}=p N /(N-p)(1<p<N)$ is the critical Sobolev exponent;

Let $J, K_{\lambda, \mu}: W \rightarrow \mathbb{R}$ be the functionals defined by

$$
\begin{aligned}
J(z) & =\int_{\Omega} h(x)|u|^{\alpha}|v|^{\beta} d x \\
K_{\lambda, \mu}(z) & =\int_{\Omega}\left(\lambda f(x)|u|^{q}+\mu g(x)|v|^{q}\right) d x
\end{aligned}
$$

for all $z=(u, v) \in W$.

As the energy functional $I_{\lambda, \mu}$ is not bounded below on $W$, it is useful to consider the functional on the Nehari manifold

$$
\mathcal{N}_{\lambda, \mu}=\left\{z \in W \backslash\{0\} \mid\left\langle I_{\lambda, \mu}^{\prime}(z), z\right\rangle=0\right\}
$$

Thus, $z=(u, v) \in \mathcal{N}_{\lambda, \mu}$ if and only if

$$
\left\langle I_{\lambda, \mu}^{\prime}(z), z\right\rangle=\|z\|^{p}-K_{\lambda, \mu}(z)-J(z)=0 .
$$

Note that $\mathcal{N}_{\lambda, \mu}$ contains every nonzero solution of System $\left(S_{\lambda f, \mu g, h}\right)$. Moreover, we have the following results

Lemma 2.1. The energy functional $I_{\lambda, \mu}$ is coercive and bounded on $\mathcal{N}_{\lambda, \mu}$.

Proof. If $z=(u, v) \in \mathcal{N}_{\lambda, \mu}$, then by (2.3), the Hölder inequality, and the Sobolev embedding theorem,

$$
I_{\lambda, \mu}(z)=\frac{\alpha+\beta-p}{p(\alpha+\beta)}\|z\|^{p}-\frac{\alpha+\beta-q}{q(\alpha+\beta)} K_{\lambda, \mu}(z)
$$


International Journal of Mathematics and Mathematical Sciences

$$
\geq \frac{\alpha+\beta-p}{p(\alpha+\beta)}\|z\|^{p}-\frac{\alpha+\beta-q}{q(\alpha+\beta)} S^{-q / p}|\Omega|^{(\alpha+\beta-q) /(\alpha+\beta)}\left(\lambda^{p /(p-q)}+\mu^{p /(p-q)}\right)^{(p-q) / p}\|z\|^{q} .
$$

Thus, $I_{\lambda, \mu}$ is coercive and bounded on $\mathcal{N}_{\lambda, \mu}$.

Define

$$
\Phi_{\lambda, \mu}(z)=\left\langle I_{\lambda, \mu}^{\prime}(z), z\right\rangle
$$

Then for $u \in \mathcal{N}_{\lambda, \mu}$,

$$
\begin{aligned}
\left\langle\Phi_{\lambda, \mu}^{\prime}(z), z\right\rangle & =p\|z\|^{p}-q K_{\lambda, \mu}(z)-(\alpha+\beta) J(z) \\
& =(p-q)\|z\|^{p}-(\alpha+\beta-q) J(z) \\
& =(\alpha+\beta-q) K_{\lambda, \mu}(z)-(\alpha+\beta-p)\|z\|^{p} .
\end{aligned}
$$

Similar to the method used in Tarantello [23], we split $\mathcal{N}_{\lambda, \mu}$ into three parts:

$$
\begin{aligned}
& \mathcal{N}_{\lambda, \mu}^{+}=\left\{z \in \mathcal{N}_{\lambda, \mu}:\left\langle\Phi_{\lambda, \mu}^{\prime}(z), z\right\rangle>0\right\}, \\
& \mathcal{N}_{\lambda, \mu}^{0}=\left\{z \in \mathcal{N}_{\lambda, \mu}:\left\langle\Phi_{\lambda, \mu}^{\prime}(z), z\right\rangle=0\right\}, \\
& \mathcal{N}_{\lambda, \mu}^{-}=\left\{z \in \mathcal{N}_{\lambda, \mu}:\left\langle\Phi_{\lambda, \mu}^{\prime}(z), z\right\rangle<0\right\} .
\end{aligned}
$$

Then, we have the following results.

Lemma 2.2. Assume that $z_{0}$ is a local minimizer for $I_{\lambda, \mu}$ on $\mathcal{N}_{\lambda, \mu}$ and $z_{0} \notin \mathcal{N}_{\lambda, \mu}^{0}$. Then $I_{\lambda, \mu}^{\prime}\left(z_{0}\right)=0$ in $W^{-1}$.

Proof. Our proof is almost the same as that in [24, Theorem 2.3].

Lemma 2.3. One has the following:

(i) if $z \in \mathcal{N}_{\lambda, \mu^{\prime}}^{+}$then $K_{\lambda, \mu}(z)>0$;

(ii) if $z \in \mathcal{N}_{\lambda, \mu^{\prime}}^{0}$ then $K_{\lambda, \mu}(z)>0$ and $J(z)>0$;

(iii) if $z \in \mathcal{N}_{\lambda, \mu^{\prime}}^{-}$then $J(z)>0$.

Proof. The proof is immediate from (2.7) and (2.8).

Moreover, we have the following result.

Lemma 2.4. If $0<\lambda^{p /(p-q)}+\mu^{p /(p-q)}<\Lambda_{1}$, then $\mathcal{N}_{\lambda, \mu}^{0}=\emptyset$ where $\Lambda_{1}$ is the same as in (1.6).

Proof. We argue by contradiction. Assume that there exist $\lambda, \mu>0$ with

$$
0<\lambda^{p /(p-q)}+\mu^{p /(p-q)}<\Lambda_{1}
$$


such that $\mathcal{N}_{\lambda, \mu}^{0} \neq \emptyset$. Then by (2.7) and (2.8), for $u \in \mathcal{N}_{\lambda, \mu^{\prime}}^{0}$ on has the following:

$$
\begin{aligned}
& \|z\|^{p}=\frac{\alpha+\beta-q}{p-q} J(z), \\
& \|z\|^{p}=\frac{\alpha+\beta-q}{\alpha+\beta-p} K_{\lambda, \mu}(z) .
\end{aligned}
$$

By $|f|_{\infty}=|g|_{\infty}=|h|_{\infty}=1$, the Hölder inequality, and the Sobolev embedding theorem, we have

$$
\begin{gathered}
\|z\| \geq\left(\frac{p-q}{\alpha+\beta-q} S^{(\alpha+\beta) / p}\right)^{1 /(\alpha+\beta-p)}, \\
\|z\| \leq\left(\frac{\alpha+\beta-q}{\alpha+\beta-p} S^{-q / p}|\Omega|^{(\alpha+\beta-q) /(\alpha+\beta)}\right)^{1 /(p-q)}\left(\lambda^{p /(p-q)}+\mu^{p(p-q)}\right)^{1 / p} .
\end{gathered}
$$

This implies

$$
\begin{gathered}
\lambda^{p /(p-q)}+\mu^{p /(p-q)} \lambda^{p /(p-q)}+\mu^{p /(p-q)} \geq\left(\frac{p-q}{\alpha+\beta-q}\right)^{p /(\alpha+\beta-p)}\left(\frac{\alpha+\beta-q}{\alpha+\beta-p}|\Omega|^{(\alpha+\beta-q) /(\alpha+\beta)}\right)^{-p /(p-q)} \\
\times S^{(\alpha+\beta) /(\alpha+\beta-p)+q /(p-q)}=\Lambda_{1}
\end{gathered}
$$

which contradicts $0<\mathcal{\lambda}^{p /(p-q)}+\mu^{p /(p-q)}<\Lambda_{1}$.

By Lemma 2.4, we write $\mathcal{N}_{\lambda, \mu}=\mathcal{N}_{\lambda, \mu}^{+} \cup \mathcal{N}_{\lambda, \mu}^{-}$and define

$$
\theta_{\lambda, \mu}=\inf _{z \in \mathcal{N}_{\lambda, \mu}} I_{\lambda, \mu}(z) ; \quad \theta_{\lambda, \mu}^{+}=\inf _{z \in \mathcal{N}_{\lambda, \mu}^{+}} I_{\lambda, \mu}(z) ; \quad \theta_{\lambda, \mu}^{-}=\inf _{z \in \mathcal{N}_{\lambda, \mu}^{-}} I_{\lambda, \mu}(z)
$$

Then we get the following result.

Theorem 2.5. (i) If $0<\lambda^{p /(p-q)}+\mu^{p /(p-q)}<\Lambda_{1}$, then one has $\theta_{\lambda, \mu} \leq \theta_{\lambda, \mu}^{+}<0$;

(ii) if $0<\lambda^{p /(p-q)}+\mu^{p /(p-q)}<(q / p)^{p /(p-q)} \Lambda_{1}$, then $\theta_{\lambda, \mu}^{-}>d_{0}$ for some positive constant $d_{0}$ depending on $\lambda, \mu, p, q, N, S,|\Omega|,|f|_{\infty},|g|_{\infty}$ and $|h|_{\infty}$.

Proof. (i) Let $z=(u, v) \in \mathcal{N}_{\lambda, \mu}^{+}$. By (2.7)

$$
\frac{p-q}{\alpha+\beta-q}\|z\|^{p}>J(z)
$$


and so

$$
\begin{aligned}
I_{\lambda, \mu}(z) & =\left(\frac{1}{p}-\frac{1}{q}\right)\|z\|^{p}+\left(\frac{1}{q}-\frac{1}{\alpha+\beta}\right) J(z) \\
& <\left[\left(\frac{1}{p}-\frac{1}{q}\right)+\left(\frac{1}{q}-\frac{1}{\alpha+\beta}\right) \frac{p-q}{\alpha+\beta-q}\right]\|z\|^{p} \\
& =-\frac{(p-q)(\alpha+\beta-p)}{p q(\alpha+\beta)}\|z\|^{p}<0 .
\end{aligned}
$$

Therefore, from the definitions of $\theta_{\lambda, \mu}, \theta_{\lambda, \mu^{\prime}}^{+}$we can deduce that $\theta_{\lambda, \mu} \leq \theta_{\lambda, \mu}^{+}<0$.

(ii) Let $z \in \mathcal{N}_{\lambda, \mu}^{-}$. By (2.7)

$$
\frac{p-q}{\alpha+\beta-q}\|z\|^{p}<J(z)
$$

Moreover, by $|h|_{\infty}=1$, the Hölder inequality, and the Sobolev embedding theorem,

$$
J(z) \leq S^{-(\alpha+\beta) / p}\|z\|^{\alpha+\beta}
$$

This implies

$$
\|z\|>\left(\frac{p-q}{\alpha+\beta-q}\right)^{1 /(\alpha+\beta-p)} S^{(\alpha+\beta) / p(\alpha+\beta-p)} \quad \forall z \in \mathcal{N}_{\lambda, \mu}^{-} .
$$

By (2.5), one has the following:

$$
\begin{aligned}
I_{\lambda, \mu}(z) \geq & \|z\|^{q}\left[\frac{\alpha+\beta-p}{p(\alpha+\beta)}\|z\|^{p-q}-\frac{\alpha+\beta-q}{q(\alpha+\beta)} S^{-q / p}|\Omega|^{(\alpha+\beta-q) /(\alpha+\beta)}\left(\lambda^{p /(p-q)}+\mu^{p /(p-q)}\right)^{(p-q) / p}\right] \\
> & \left(\frac{p-q}{\alpha+\beta-q}\right)^{q /(\alpha+\beta-p)} S^{q(\alpha+\beta) / p(\alpha+\beta-p)} \\
& \times\left[\frac{\alpha+\beta-p}{p(\alpha+\beta)} S^{(p-q)(\alpha+\beta) / p(\alpha+\beta-p)}\left(\frac{p-q}{\alpha+\beta-q}\right)^{p-q /(\alpha+\beta-p)}\right. \\
& \left.\left.-\frac{\alpha+\beta-q}{q(\alpha+\beta)} S^{-q / p}|\Omega|^{(\alpha+\beta-q) /(\alpha+\beta)}\left(\lambda^{p /(p-q)}+\mu^{p /(p-q)}\right)\right)^{(p-q) / p}\right] .
\end{aligned}
$$

Thus, if $0<\lambda^{p /(p-q)}+\mu^{p /(p-q)}<(q / p)^{p /(p-q)} \Lambda_{1}$, then

$$
I_{\lambda, \mu}(z)>d_{0} \quad \forall z \in \mathcal{N}_{\lambda, \mu^{\prime}}^{-}
$$

for some positive constant $d_{0}=d_{0}\left(\lambda, \mu, p, q, N, S,|\Omega|,|f|_{\infty^{\prime}}|g|_{\infty},|h|_{\infty}\right)$. This completes the proof. 
For each $z \in W$ with $J(z)>0$, we write

$$
t_{\max }=\left(\frac{(p-q)\|z\|^{p}}{(\alpha+\beta-q) J(z)}\right)^{1 /(\alpha+\beta-p)}>0 .
$$

Then the following lemma holds.

Lemma 2.6. Let $\lambda^{p /(p-q)}+\mu^{p /(p-q)} \in\left(0, \Lambda_{1}\right)$. For each $z \in W$ with $J(z)>0$, one has the following:

(i) if $K_{\lambda, \mu}(z) \leq 0$, then there exists a unique $t^{-}>t_{\max }$ such that $t^{-} z \in \mathcal{N}_{\lambda, \mu}^{-}$and

$$
I_{\lambda, \mu}\left(t^{-} z\right)=\sup _{t \geq 0} I_{\lambda, \mu}(t z)
$$

(ii) if $K_{\lambda, \mu}(z)>0$, then there exist unique $0<t^{+}<t_{\max }<t^{-}$such that $t^{+} z \in \mathcal{N}_{\lambda, \mu^{\prime}}^{+} t^{-} z \in \mathcal{N}_{\lambda, \mu}^{-}$ and

$$
I_{\lambda, \mu}\left(t^{+} z\right)=\inf _{0 \leq t \leq t_{\max }} I_{\lambda, \mu}(t z) ; \quad I_{\lambda, \mu}\left(t^{-} z\right)=\sup _{t \geq 0} I_{\lambda, \mu}(t z)
$$

Proof. The proof is almost the same as that in [25, Lemma 2.6] and is omitted here.

For each $z \in W$ with $K_{\lambda, \mu}(z)>0$, we write

$$
\bar{t}_{\max }=\left(\frac{(\alpha+\beta-q) K_{\lambda, \mu}(z)}{(\alpha+\beta-p)\|z\|^{p}}\right)^{1 /(p-q)}>0 .
$$

Then we have the following lemma.

Lemma 2.7. Let $\lambda^{p /(p-q)}+\mu^{p /(p-q)} \in\left(0, \Lambda_{1}\right)$. For each $z \in W$ with $K_{\lambda, \mu}(z)>0$, one has the following:

(i) if $J(z) \leq 0$, then there exists a unique $0<t^{+}<\bar{t}_{\max }$ such that $t^{+} z \in \mathcal{N}_{\lambda, \mu}^{+}$and

$$
I_{\lambda, \mu}\left(t^{+} z\right)=\inf _{t \geq 0} I_{\lambda, \mu}(t z)
$$

(ii) if $J(z)>0$, then there exist unique $0<t^{+}<\bar{t}_{\max }<t^{-}$such that $t^{+} z \in \mathcal{N}_{\lambda, \mu^{+}}^{+} t^{-} z \in \mathcal{N}_{\lambda, \mu}^{-}$ and

$$
I_{\lambda, \mu}\left(t^{+} z\right)=\inf _{0 \leq t \leq t_{\max }} I_{\lambda, \mu}(t z) ; \quad I_{\lambda, \mu}\left(t^{-} z\right)=\sup _{t \geq 0} I_{\lambda, \mu}(t z)
$$

Proof. The proof is almost the same as that in [25, Lemma 2.7] and is omitted here. 
International Journal of Mathematics and Mathematical Sciences

\section{Proofs of Theorems 1.1 and 1.2}

First, we give the following definitions about $(\mathrm{PS})_{c}$-sequence.

Definition 3.1. Let $c \in \mathbb{R}, W$ be a Banach space and $I \in C^{1}(W, \mathbb{R})$.

(i) $\left\{z_{n}\right\}$ is a (PS) $)_{c}$-sequence in $W$ for $I$ if $I\left(z_{n}\right)=c+o_{n}(1)$ and $I^{\prime}\left(z_{n}\right)=o_{n}(1)$ strongly in $W^{-1}$ as $n \rightarrow \infty$.

(ii) We say that $I$ satisfies the (PS) ${ }_{c}$ condition if any (PS) ${ }_{c}$-sequence $\left\{z_{n}\right\}$ in $W$ for $I$ has a convergent subsequence.

Now, we use the idea of Tarantello [23] to get the following results.

Proposition 3.2. Let $1 \leq q<p<N$ and $p<\alpha+\beta \leq p^{*}$, we have

(i) if $0<\lambda^{p /(p-q)}+\mu^{p /(p-q)}<\Lambda_{1}$, then there exists a $(P S)_{\theta_{\lambda, \mu}}$-sequence $\left\{z_{n}\right\} \subset \mathcal{N}_{\lambda, \mu}$ in $W$ for $I_{\lambda, \mu} ;$

(ii) if $0<\lambda^{p /(p-q)}+\mu^{p /(p-q)}<(q / p)^{p /(p-q)} \Lambda_{1}$, then there exists a $(P S)_{\theta_{\lambda_{1}^{-},}}$-sequence $\left\{z_{n}\right\} \subset$ $N_{\lambda, \mu}$ in $W$ for $I_{\lambda, \mu}$

where $\Lambda_{1}$ is the positive constant given in (1.6).

Proof. The proof is almost the same as that in [8, Proposition 9].

Now, we establish the existence of a local minimum for $I_{\lambda, \mu}$ on $\mathcal{N}_{\lambda, \mu}^{+}$.

Theorem 3.3. Let $\Lambda_{1}$ be the same positive constant as in (1.6). If $1 \leq q<p<N, p<\alpha+\beta \leq p^{*}$, and $0<\lambda^{p /(p-q)}+\mu^{p /(p-q)}<\Lambda_{1}$, then $I_{\lambda, \mu}$ has a minimizer $z_{\lambda, \mu}^{1}$ in $N_{\lambda, \mu}^{+}$and it satisfies the following:

(i) $I_{\lambda, \mu}\left(z_{\lambda, \mu}^{1}\right)=\theta_{\lambda, \mu}=\theta_{\lambda, \mu}^{+}<0$;

(ii) $z_{\lambda, \mu}^{1}$ is a positive solution of System $\left(S_{\lambda f, \mu g, h}\right)$;

(iii) $I_{\lambda, \mu}\left(z_{\lambda, \mu}^{1}\right) \rightarrow 0$ as $\lambda \rightarrow 0^{+}, \mu \rightarrow 0^{+}$.

Proof. By Proposition $3.2(i)$, there exists a minimizing sequence $\left\{z_{n}\right\}$ for $I_{\lambda, \mu}$ on $\mathcal{N}_{\lambda, \mu}$ such that

$$
I_{\lambda, \mu}\left(z_{n}\right)=\theta_{\lambda, \mu}+o_{n}(1), \quad I_{\lambda, \mu}^{\prime}\left(z_{n}\right)=o_{n}(1) \quad \text { in } W^{-1}
$$

Since $I_{\lambda, \mu}$ is coercive on $\mathcal{N}_{\lambda, \mu}$ (see Lemma 2.1), we get that $\left\{z_{n}\right\}$ is bounded in $W$. Then there exist a subsequence $\left\{z_{n}=\left(u_{n}, v_{n}\right)\right\}$ and $z_{\lambda, \mu}^{1}=\left(u_{\lambda, \mu}^{1}, v_{\lambda, \mu}^{1}\right) \in W$ such that

$$
\begin{gathered}
u_{n} \rightarrow u_{\lambda, \mu^{\prime}}^{1} \quad v_{n} \rightarrow v_{\lambda, \mu}^{1} \quad \text { weakly in } W_{0}^{1, p}(\Omega), \\
u_{n} \longrightarrow u_{\lambda, \mu^{\prime}}^{1} \quad v_{n} \longrightarrow v_{\lambda, \mu}^{1} \quad \text { almost everywhere in } \Omega, \\
u_{n} \longrightarrow u_{\lambda, \mu^{\prime}}^{1} \quad v_{n} \longrightarrow v_{\lambda, \mu}^{1} \quad \text { strongly in } L^{S}(\Omega) \forall 1 \leq s<p^{*} .
\end{gathered}
$$


This implies

$$
K_{\lambda, \mu}\left(z_{n}\right)=K_{\lambda, \mu}\left(z_{\lambda, \mu}^{1}\right)+o_{n}(1) \quad \text { as } n \longrightarrow \infty
$$

First, we claim that $z_{\lambda, \mu}^{1}$ is a nontrivial solution of System $\left(S_{\lambda f, \mu g, h}\right)$. By (3.1) and (3.2), it is easy to verify that $z_{\lambda, \mu}^{1}$ is a weak solution of System $\left(S_{\lambda f, \mu g, h}\right)$. From $z_{n} \in \mathcal{N}_{\lambda, \mu}$ and (2.4), we deduce that

$$
K_{\lambda, \mu}\left(z_{n}\right)=\frac{q(\alpha+\beta-p)}{p(\alpha+\beta-q)}\left\|z_{n}\right\|^{p}-\frac{q(\alpha+\beta)}{\alpha+\beta-q} I_{\lambda, \mu}\left(z_{n}\right)
$$

Let $n \rightarrow \infty$ in (3.4), by (3.1), (3.3), and $\theta_{\lambda, \mu}<0$, we get

$$
K_{\lambda, \mu}\left(z_{\lambda, \mu}^{1}\right) \geq-\frac{q(\alpha+\beta)}{\alpha+\beta-q} \theta_{\lambda, \mu}>0
$$

Thus, $z_{\lambda, \mu}^{1} \in \mathcal{N}_{\lambda, \mu}$ is a nontrivial solution of System $\left(S_{\lambda f, \mu g, h}\right)$. Now we prove that $z_{n} \rightarrow z_{\lambda, \mu}^{1}$ strongly in $W$ and $I_{\lambda, \mu}\left(z_{\lambda, \mu}^{1}\right)=\theta_{\lambda, \mu}$. By (3.4), if $z \in \mathcal{N}_{\lambda, \mu}$, then

$$
I_{\lambda, \mu}(z)=\frac{\alpha+\beta-p}{p(\alpha+\beta)}\|z\|^{p}-\frac{\alpha+\beta-q}{q(\alpha+\beta)} K_{\lambda, \mu}(z)
$$

In order to prove that $I_{\lambda, \mu}\left(z_{\lambda, \mu}^{1}\right)=\theta_{\lambda, \mu}$, it suffices to recall that $z_{\lambda, \mu}^{1} \in \mathcal{N}_{\lambda, \mu}$, by (3.6), and apply Fatou's lemma to get

$$
\begin{aligned}
\theta_{\lambda, \mu} & \leq I_{\lambda, \mu}\left(z_{\lambda, \mu}^{1}\right)=\frac{\alpha+\beta-p}{p(\alpha+\beta)}\left\|z_{\lambda, \mu}^{1}\right\|^{p}-\frac{\alpha+\beta-q}{q(\alpha+\beta)} K_{\lambda, \mu}\left(z_{\lambda, \mu}^{1}\right) \\
& \leq \liminf _{n \rightarrow \infty}\left(\frac{\alpha+\beta-p}{p(\alpha+\beta)}\left\|z_{n}\right\|^{p}-\frac{\alpha+\beta-q}{q(\alpha+\beta)} K_{\lambda, \mu}\left(z_{n}\right)\right) \\
& \leq \liminf _{n \rightarrow \infty} I_{\lambda, \mu}\left(z_{n}\right)=\theta_{\lambda, \mu} .
\end{aligned}
$$

This implies that $I_{\lambda, \mu}\left(z_{\lambda, \mu}^{1}\right)=\theta_{\lambda, \mu}$ and $\lim _{n \rightarrow \infty}\left\|z_{n}\right\|^{p}=\left\|z_{\lambda, \mu}^{1}\right\|^{p}$. Let $\widetilde{z}_{n}=z_{n}-z_{\lambda, \mu^{\prime}}^{1}$, then by Brézis and Lieb lemma [26] implies

$$
\left\|\tilde{z}_{n}\right\|^{p}=\left\|z_{n}\right\|^{p}-\left\|z_{\lambda, \mu}^{1}\right\|^{p}
$$

Therefore, $z_{n} \rightarrow z_{\lambda, \mu}^{1}$ strongly in $W$. Moreover, we have $z_{\lambda, \mu}^{1} \in \mathcal{N}_{\lambda, \mu}^{+}$. Thus $\theta_{\lambda, \mu}=\theta_{\lambda, \mu}^{+}$. On the contrary, if $z_{\lambda, \mu}^{1} \in \mathcal{N}_{\lambda, \mu}^{-}$, then by (2.17), (3.5), we have that $J\left(z_{\lambda, \mu}^{1}\right)>0$ and $K_{\lambda, \mu}\left(z_{\lambda, \mu}^{1}\right)>0$. Thus, 
from Lemma 2.6 (ii), there exist unique $t_{1}^{+}$and $t_{1}^{-}$such that $t_{1}^{+} z_{\lambda, \mu}^{1} \in \mathcal{N}_{\lambda, \mu}^{+}$and $t_{1}^{-} z_{\lambda, \mu}^{1} \in \mathcal{N}_{\lambda, \mu}^{-}$. In particular, we have $t_{1}^{+}<t_{1}^{-}=1$. Since

$$
\frac{d}{d t} I_{\lambda, \mu}\left(t_{1}^{+} z_{\lambda, \mu}^{1}\right)=0, \quad \frac{d^{2}}{d t^{2}} I_{\lambda, \mu}\left(t_{1}^{+} z_{\lambda, \mu}^{1}\right)>0,
$$

there exists $t_{1}^{+}<\bar{t} \leq t_{1}^{-}$such that $I_{\lambda, \mu}\left(t_{1}^{+} z_{\lambda, \mu}^{1}\right)<I_{\lambda, \mu}\left(\bar{t} z_{\lambda, \mu}^{1}\right)$. By Lemma 2.6,

$$
I_{\lambda, \mu}\left(t_{1}^{+} z_{\lambda, \mu}^{1}\right)<I_{\lambda, \mu}\left(\bar{t} z_{\lambda, \mu}^{1}\right) \leq I_{\lambda, \mu}\left(t_{1}^{-} z_{\lambda, \mu}^{1}\right)=I_{\lambda, \mu}\left(z_{\lambda, \mu}^{1}\right)=\theta_{\lambda, \mu}
$$

which is a contradiction. Since $I_{\lambda, \mu}\left(z_{\lambda, \mu}^{1}\right)=I_{\lambda, \mu}\left(\left|z_{\lambda, \mu}^{1}\right|\right)$ and $\left|z_{\lambda, \mu}^{1}\right| \in \mathcal{N}_{\lambda, \mu^{\prime}}^{+}$by Lemma 2.2 we may assume that $z_{\lambda, \mu}^{1}$ is a nontrivial nonnegative solution of System $\left(S_{\lambda f, \mu g, h}\right)$.

In particular $u_{\lambda, \mu}^{1} \not \equiv 0, v_{\lambda, \mu}^{1} \not \equiv 0$. Indeed, without loss of generality, we may assume that $v_{\lambda, \mu}^{1} \equiv 0$. Then as $u_{\lambda, \mu}^{1}$ is a nontrivial nonnegative solution of

$$
\begin{gathered}
-\Delta_{p} u=\lambda f(x)|u|^{q-2} u \quad \text { in } \Omega, \\
u=0 \quad \text { on } \partial \Omega .
\end{gathered}
$$

By the standard regularity theory, we have $u_{\lambda, \mu}^{1}>0$ in $\Omega$ and

$$
\left\|\left(u_{\lambda, \mu^{\prime}}^{1} 0\right)\right\|^{p}=K_{\lambda, \mu}\left(u_{\lambda, \mu^{\prime}}^{1} 0\right)>0
$$

Moreover, by conditions $(A 1),(A 2)$ and $u_{\lambda, \mu}^{1}>0$ in $\Omega$, we may choose $w \in W_{0}^{1, p}(\Omega) \backslash\{0\}$ such that

$$
\begin{gathered}
\|(0, w)\|^{p}=K_{\lambda, \mu}(0, w)>0, \\
J\left(u_{\lambda, \mu}^{1}, w\right) \geq 0 .
\end{gathered}
$$

Now

$$
K_{\lambda, \mu}\left(u_{\lambda, \mu^{\prime}}^{1}, w\right)=K_{\lambda, \mu}\left(u_{\lambda, \mu^{\prime}}^{1} 0\right)+K_{\lambda, \mu}(0, w)>0
$$

and so by Lemma 2.7 there is unique $0<t^{+}<\bar{t}_{\max }$ such that $\left(t^{+} u_{\lambda, \mu^{\prime}}^{1} t^{+} w\right) \in \mathcal{N}_{\lambda, \mu}^{+}$. Moreover,

$$
\begin{aligned}
\bar{t}_{\max }= & \left(\frac{(\alpha+\beta-q) K_{\lambda, \mu}\left(u_{\lambda, \mu^{\prime}}^{1} w\right)}{(\alpha+\beta-p)\left\|\left(u_{\lambda, \mu^{\prime}}^{1} w\right)\right\|^{p}}\right)=\left(\frac{\alpha+\beta-q}{\alpha+\beta-p}\right)>1, \\
& I_{\lambda, \mu}\left(t^{+} u_{\lambda, \mu^{\prime}}^{1} t^{+} w\right)=\inf _{0 \leq t \leq \bar{t}_{\max }} I_{\lambda, \mu}\left(t u_{\lambda, \mu^{\prime}}^{1} t w\right) .
\end{aligned}
$$


This implies

$$
\theta_{\lambda, \mu}^{+} \leq I_{\lambda, \mu}\left(t^{+} u_{\lambda, \mu^{\prime}}^{1} t^{+} w\right) \leq I_{\lambda, \mu}\left(u_{\lambda, \mu^{\prime}}^{1} w\right)<I_{\lambda, \mu}\left(u_{\lambda, \mu^{\prime}}^{1} 0\right)=\theta_{\lambda, \mu}^{+}
$$

which is a contradiction.

By a standard bootstrap argument, it is proved that a weak solution for System $\left(S_{\lambda f, \mu g, h}\right)$ is in the space $C^{2}(\bar{\Omega}) \times C^{2}(\bar{\Omega})$, and it is really a classical solution. Finally, by the Harnack inequality [27] we deduce that $z_{\lambda, \mu}^{1}$ is a positive solution of System $\left(S_{\lambda f, \mu g, h}\right)$. Moreover, by Theorem $2.5(i)$ and (2.5) we have

$$
0>\theta_{\lambda, \mu}^{+}=I_{\lambda, \mu}\left(z_{\lambda, \mu}^{1}\right)>-\frac{\alpha+\beta-q}{q(\alpha+\beta)} S^{-q / p}|\Omega|^{(\alpha+\beta-q) /(\alpha+\beta)}\left(\lambda^{p /(p-q)}+\mu^{p /(p-q)}\right)^{(p-q) / p}\left\|z_{\lambda, \mu}^{1}\right\|^{q} .
$$

This implies that $I_{\lambda, \mu}\left(z_{\lambda, \mu}^{1}\right) \rightarrow 0$ as $\lambda \rightarrow 0^{+}, \mu \rightarrow 0^{+}$.

Next, we establish the existence of a local minimum for $I_{\lambda, \mu}$ on $\mathcal{N}_{\lambda, \mu}^{-}$in the subcritical case $p<\alpha+\beta<p^{*}$. This implies that there exists the second positive solution in the subcritical case $p<\alpha+\beta<p^{*}$.

Theorem 3.4. Let $\Lambda_{1}$ be the same positive constant as in (1.6). If $1 \leq q<p<N, p<\alpha+\beta<p^{*}$, and $0<\lambda^{p /(p-q)}+\mu^{p /(p-q)}<(q / p)^{p /(p-q)} \Lambda_{1}$, then $I_{\lambda, \mu}$ has a minimizer $z_{\lambda, \mu}^{2}$ in $N_{\lambda, \mu}^{-}$and it satisfies the following:

(i) $I_{\lambda, \mu}\left(z_{\lambda, \mu}^{2}\right)=\theta_{\lambda, \mu^{\prime}}^{-}$

(ii) $z_{\lambda, \mu}^{2}$ is a positive solution of System $\left(S_{\lambda f, \mu g, h}\right)$;

Proof. Let $\left\{z_{n}\right\}$ be a minimizing sequence for $I_{\lambda, \mu}$ on $\mathcal{N}_{\lambda, \mu}^{-}$. Then by $I_{\lambda, \mu}$ coercive on $\mathcal{N}_{\lambda, \mu}$ and the compact imbedding theorem, there exist a subsequence $\left\{z_{n}=\left(u_{n}, v_{n}\right)\right\}$ and $z_{\lambda, \mu}^{2}=$ $\left(u_{\lambda, \mu}^{2}, v_{\lambda, \mu}^{2}\right) \in W$ such that

$$
\begin{gathered}
u_{n} \rightarrow u_{\lambda, \mu^{\prime}}^{2} v_{n} \rightarrow v_{\lambda, \mu}^{2} \quad \text { weakly in } W_{0}^{1, p}(\Omega), \\
u_{n} \longrightarrow u_{\lambda, \mu^{\prime}}^{2} v_{n} \longrightarrow v_{\lambda, \mu}^{2} \quad \text { strongly in } L^{q}(\Omega), L^{\alpha+\beta}(\Omega) .
\end{gathered}
$$

This implies

$$
K_{\lambda, \mu}\left(z_{n}\right)=K_{\lambda, \mu}\left(z_{\lambda, \mu}^{2}\right)+o_{n}(1), \quad J\left(z_{n}\right)=J\left(z_{\lambda, \mu}^{2}\right)+o_{n}(1)
$$

By (2.17) and (2.19) there exists a positive number $\bar{C}$ such that

$$
J\left(z_{n}\right)>\bar{C}
$$


This implies

$$
J\left(z_{\lambda, \mu}^{2}\right) \geq \bar{C}
$$

Now, we prove that $\mathrm{z}_{n} \rightarrow z_{\lambda, \mu}^{2}$ strongly in $W$. Suppose otherwise, then $\left\|z_{\lambda, \mu}^{2}\right\|<$ $\lim \inf _{n \rightarrow \infty}\left\|z_{n}\right\|$. By Lemma 2.6, there exists a unique $t_{2}^{-}$such that $t_{2}^{-} z_{\lambda, \mu}^{2} \in \mathcal{N}_{\lambda, \mu}^{-}$. Since $z_{n} \in \mathcal{N}_{\lambda, \mu^{\prime}}^{-} I_{\lambda, \mu}\left(z_{n}\right) \geq I_{\lambda, \mu}\left(t z_{n}\right)$ for all $t \geq 0$, we have

$$
\theta_{\lambda, \mu}^{-} \leq I_{\lambda, \mu}\left(t^{-} z_{\lambda, \mu}^{2}\right)<\lim _{n \rightarrow \infty} I_{\lambda, \mu}\left(t^{-} z_{n}\right) \leq \lim _{n \rightarrow \infty} I_{\lambda, \mu}\left(z_{n}\right)=\theta_{\lambda, \mu^{\prime}}^{-}
$$

and this is a contradiction. Hence

$$
z_{n} \longrightarrow z_{\lambda, \mu}^{2} \quad \text { strongly in } W \text {. }
$$

This implies

$$
I_{\lambda, \mu}\left(z_{\lambda, \mu}^{2}\right)=\lim _{n \rightarrow \infty} I_{\lambda, \mu}\left(z_{n}\right)=\theta_{\lambda, \mu}^{-}
$$

Since $I_{\lambda, \mu}\left(z_{\lambda, \mu}^{2}\right)=I_{\lambda, \mu}\left(\left|z_{\lambda, \mu}^{2}\right|\right)$ and $\left|z_{\lambda, \mu}^{2}\right| \in \mathcal{N}_{\lambda, \mu^{\prime}}^{-}$by Lemma 2.2 and (3.21) we may assume $z_{\lambda, \mu}^{2}$ is a nontrivial nonnegative solution of System $\left(S_{\lambda f, \mu g, h}\right)$. Finally, by using the same arguments as in the proof of Theorem 3.3, for all $0<\lambda^{p /(p-q)}+\mu^{p /(p-q)}<(q / p)^{p /(p-q)} \Lambda_{1}$, we have that $z_{\lambda, \mu}^{2}$ is a positive solution of System $\left(S_{\lambda f, \mu g, h}\right)$.

Now, we complete the proofs of Theorems 1.1 and 1.2: Theorem 1.1 follows from Theorem 3.3. By Theorems 3.3 and 3.4, we obtain that for all $1 \leq q<p<N, p<\alpha+\beta<p^{*}$, $\lambda, \mu>0$, and $0<\lambda^{p /(p-q)}+\mu^{p /(p-q)}<(q / p)^{p /(p-q)} \Lambda_{1}$. System $\left(S_{\lambda f, \mu g, h}\right)$ has two positive solutions $z_{\lambda, \mu^{\prime}}^{1} z_{\lambda, \mu}^{2}$ with $z_{\lambda, \mu}^{1} \in \mathcal{N}_{\lambda, \mu^{\prime}}^{+} z_{\lambda, \mu}^{2} \in \mathcal{N}_{\lambda, \mu}^{-}$. Since $\mathcal{N}_{\lambda, \mu}^{+} \cap \mathcal{N}_{\lambda, \mu}^{-}=\emptyset$, this implies that $z_{\lambda, \mu}^{1}$ and $z_{\lambda, \mu}^{2}$ are distinct. This completes the proofs of Theorems 1.1 and 1.2.

\section{Proof of Theorem 1.3}

For the existence of a second positive solution of System $\left(S_{\lambda f, \mu g, h}\right)$ in the critical case $\alpha+\beta=p^{*}$, we will however need here a stronger restriction on $h(x)$, namely, $h(x)>0$ in $\Omega$ but $f(x)$ and $g(x)$ may be also allowed to change sign in $\Omega$. Now, we will establish the existence of a local minimum for $I_{\lambda, \mu}$ on $\mathcal{N}_{\lambda, \mu}^{-}$in the critical case $\alpha+\beta=p^{*}$ to obtain a second positive solution of System $\left(S_{\lambda f, \mu g, h}\right)$.

Lemma 4.1. If $\left\{z_{n}\right\} \subset W$ is a $(P S)_{c}$-sequence for $I_{\lambda, \mu}$, then $\left\{z_{n}\right\}$ is bounded in $W$.

Proof. Let $z_{n}=\left(u_{n}, v_{n}\right)$. We argue by contradiction. Assume that $\left\|z_{n}\right\| \rightarrow \infty$. Let

$$
\widehat{z}_{n}=\left(\widehat{u}_{n}, \widehat{v}_{n}\right)=\frac{z_{n}}{\left\|z_{n}\right\|}=\left(\frac{u_{n}}{\left\|z_{n}\right\|}, \frac{v_{n}}{\left\|z_{n}\right\|}\right) .
$$


We may assume that $\widehat{z}_{n} \rightarrow \widehat{z}=(\widehat{u}, \widehat{v})$ in $W$. This implies that $\widehat{u}_{n} \rightarrow \widehat{u}, \widehat{v}_{n} \rightarrow \widehat{v}$ strongly in $L^{s}(\Omega)$ for all $1 \leq s<p^{*}$ and

$$
K_{\lambda, \mu}\left(\widehat{z}_{n}\right)=K_{\lambda, \mu}(\widehat{z})+o_{n}(1) .
$$

Since $\left\{z_{n}\right\}$ is a (PS) $)_{c}$-sequence for $I_{\lambda, \mu}$ and $\left\|z_{n}\right\| \rightarrow \infty$, there hold

$$
\begin{gathered}
\frac{1}{p}\left\|\widehat{z}_{n}\right\|^{p}-\frac{\left\|z_{n}\right\|^{q-p}}{q} K_{\lambda, \mu}\left(\widehat{z}_{n}\right)-\frac{\left\|z_{n}\right\|^{\alpha+\beta-p}}{\alpha+\beta} J\left(\widehat{z}_{n}\right)=o_{n}(1), \\
\left\|\widehat{z}_{n}\right\|^{p}-\left\|z_{n}\right\|^{q-p} K_{\lambda, \mu}\left(\widehat{z}_{n}\right)-\left\|z_{n}\right\|^{\alpha+\beta-p} J\left(\widehat{z}_{n}\right)=o_{n}(1) .
\end{gathered}
$$

From (4.2)-(4.4), we can deduce that

$$
\left\|\widehat{z}_{n}\right\|^{p}=\frac{p(\alpha+\beta-q)}{q(\alpha+\beta-p)}\left\|z_{n}\right\|^{q-p} K_{\lambda, \mu}\left(\widehat{z}_{n}\right)+o_{n}(1)
$$

Since $1 \leq q<p$ and $\left\|z_{n}\right\| \rightarrow \infty$, (4.5) implies

$$
\left\|\widehat{z}_{n}\right\|^{p} \longrightarrow 0, \quad \text { as } n \longrightarrow \infty,
$$

which is contrary to the fact $\left\|\widehat{z}_{n}\right\|=1$.

Denote

$$
S_{\alpha, \beta}=\inf _{u, v \in W_{0}^{1, p}(\Omega) \backslash\{0\}} \frac{\int_{\Omega}\left(|\nabla u|^{p}+|\nabla v|^{p}\right) d x}{\left(\int_{\Omega}|u|^{\alpha}|v|^{\beta} d x\right)^{p /(\alpha+\beta)}} .
$$

Modifying the proof of Alves et al. [11, Theorem 5], we can easily deduce that

$$
S_{\alpha, \beta}=\left(\left(\frac{\alpha}{\beta}\right)^{\beta /(\alpha+\beta)}+\left(\frac{\beta}{\alpha}\right)^{\alpha /(\alpha+\beta)}\right) S,
$$

where $S$ is the best constant defined by

$$
S=\inf _{u \in W_{0}^{1, p}(\Omega) \backslash\{0\}} \frac{\int_{\Omega}|\nabla u|^{p} d x}{\left(\int_{\Omega}|u|^{\alpha+\beta} d x\right)^{p /(\alpha+\beta)}} .
$$

When $\alpha+\beta=p^{*}$, it is well known that $S$ is achieved if and only if $\Omega=\mathbb{R}^{N}$ by the function 


$$
U_{\varepsilon}(x)=C_{N}\left(\frac{\varepsilon^{1 / p}}{\varepsilon+|x|^{p /(p-1)}}\right)^{(N-p) / p}, \quad \varepsilon>0,
$$

where $C_{N}=\left[((N-p) /(p-1))^{p-1} N\right]^{(N-p) / p^{2}}$.

Lemma 4.2. Let $c_{\infty}=(1 / N) S_{\alpha, \beta}^{N / p}|h|_{\infty}^{-(N-p) / p}$. If $\left\{z_{n}\right\} \subset W$ is a $(P S)_{c}$-sequence for $I_{\lambda, \mu}$ with $c \in$ $\left(0, c_{\infty}\right)$, then there exists a subsequence of $\left\{z_{n}\right\}$ converging weakly to a nontrivial solution of System $\left(S_{\lambda f, \mu g, h}\right)$.

Proof. Let $\left\{z_{n}\right\} \subset W$ be a (PS) $)_{c}$-sequence for $I_{\lambda, \mu}$ with $c \in\left(0, c_{\infty}\right)$. Write $z_{n}=\left(u_{n}, v_{n}\right)$. We know from Lemma 4.1 that $\left\{z_{n}\right\}$ is bounded in $W$, and then $z_{n} \rightarrow z=(u, v)$ up to a subsequence, when $z$ is a critical point of $I_{\lambda, \mu}$. Furthermore, we may assume $u_{n} \rightarrow u, v_{n} \rightarrow v$ in $W_{0}^{1, p}(\Omega)$ and $u_{n} \rightarrow u, v_{n} \rightarrow v$ in $L^{s}(\Omega)$ for all $1 \leq s<p^{*}$ and $u_{n} \rightarrow u, v_{n} \rightarrow v$ a.e. on $\Omega$. Hence we have that $I_{\lambda, \mu}^{\prime}(z)=0$ and

$$
K_{\lambda, \mu}\left(z_{n}\right)=K_{\lambda, \mu}(z)+o_{n}(1) .
$$

Next we verify that $u \neq 0$ or $v \neq 0$. Arguing by contradiction, we assume $u \equiv 0$ and $v \equiv 0$. Set

$$
l=\lim _{n \rightarrow \infty} J\left(z_{n}\right)
$$

Since $I_{\lambda, \mu}^{\prime}\left(z_{n}\right)=o_{n}(1)$ and $\left\{z_{n}\right\}$ is bounded in $W$, then by (4.11), we can deduce that

$$
0=\left\langle\lim _{n \rightarrow \infty} I_{\lambda, \mu}^{\prime}\left(z_{n}\right), z_{n}\right\rangle=\lim _{n \rightarrow \infty}\left(\left\|z_{n}\right\|^{p}-J\left(z_{n}\right)\right)=\lim _{n \rightarrow \infty}\left\|z_{n}\right\|^{p}-l
$$

that is,

$$
\lim _{n \rightarrow \infty}\left\|z_{n}\right\|^{p}=l
$$

If $l=0$, then we get $c=\lim _{n \rightarrow \infty} I_{\lambda, \mu}\left(z_{n}\right)=0$, which contradicts with $c>0$. Thus we conclude that $l>0$. By (4.7) and (4.12), we obtain

$$
\begin{aligned}
S_{\alpha, \beta} l^{p / p^{*}} & =S_{\alpha, \beta} \lim _{n \rightarrow \infty}\left(\int_{\Omega} h(x)\left|u_{n}\right|^{\alpha}\left|v_{n}\right|^{\beta} d x\right)^{p / p^{*}} \\
& \leq S_{\alpha, \beta} \lim _{n \rightarrow \infty}\left(\int_{\Omega}|h|_{\infty}\left|u_{n}\right|^{\alpha}\left|v_{n}\right|^{\beta} d x\right)^{p / p^{*}} \\
& \leq|h|_{\infty}^{p / p^{*}} \lim _{n \rightarrow \infty}\left\|z_{n}\right\|^{p} \\
& =|h|_{\infty}^{p / p^{*}} l,
\end{aligned}
$$


which implies that

$$
l \geq S_{\alpha, \beta}^{N / p}|h|_{\infty}^{-(N-p) / p}
$$

Hence, from (4.11)-(4.16) we get

$$
\begin{aligned}
c & =\lim _{n \rightarrow \infty} I_{\lambda, \mu}\left(z_{n}\right) \\
& =\lim _{n \rightarrow \infty}\left(\frac{1}{p}\left\|z_{n}\right\|^{p}-\frac{1}{q} K_{\lambda, \mu}\left(z_{n}\right)-\frac{1}{\alpha+\beta} J\left(z_{n}\right)\right) \\
& =\left(\frac{1}{p}-\frac{1}{\alpha+\beta}\right) l \\
& \geq \frac{1}{N} S_{\alpha, \beta}^{N / p}|h|_{\infty}^{-(N-p) / p}=c_{\infty} .
\end{aligned}
$$

This is a contradiction to $c<c_{\infty}$. Therefore $z$ is a nontrivial solution of System $\left(S_{\lambda f, \mu g, h}\right)$.

Lemma 4.3. Assume that $(A 1)-(A 4)$ hold. Then for any $\lambda, \mu>0$, there exist a nonnegative function $z_{\lambda, \mu} \in W \backslash\{0\}$ such that

$$
\sup _{t \geq 0} I_{\lambda, \mu}\left(t z_{\lambda, \mu}\right)<c_{\infty}
$$

where $c_{\infty}$ is the constant given in Lemma 4.2.

In particular, $\theta_{\lambda, \mu}^{-}<c_{\infty}$ for all $0<\lambda^{p /(p-q)}+\mu^{p /(p-q)}<\Lambda_{1}$, where $\Lambda_{1}$ is as in (1.6).

Proof. From (A4), we know that there exists $0<\rho_{0} \leq r_{0}$ such that, for all $x \in B\left(0,2 \rho_{0}\right)$,

$$
h(x)=h(0)+o\left(|x|^{\delta_{0}}\right) \quad \text { for some } \delta_{0}>\frac{N}{p-1} .
$$

Now, we consider the functional $Q: W \rightarrow \mathbb{R}$ defined by

$$
Q(z)=\frac{1}{p}\|z\|^{p}-\frac{1}{\alpha+\beta} J(z) \quad \forall z=(u, v) \in W
$$

and define a cut-off function $\eta(x) \in C_{0}^{\infty}(\Omega)$ such that $\eta(x)=1$ for $|x|<\rho_{0}, \eta(x)=0$ for $|x|>2 \rho_{0}, 0 \leq \eta \leq 1$, and $|\nabla \eta| \leq C$. For $\varepsilon>0$, let

$$
u_{\varepsilon}(x)=\frac{\varepsilon^{(N-p) / p^{2}} \eta(x)}{\left(\varepsilon+|x|^{p /(p-1)}\right)^{(N-p) / p}} .
$$

From Hsu [7, Lemma 4.3], we have

$$
\left|\nabla u_{\varepsilon}\right|_{p}^{p}=\int_{\mathbb{R}^{N}}|\nabla U|^{p} d x+O\left(\varepsilon^{(N-p) / p}\right)
$$


International Journal of Mathematics and Mathematical Sciences

$$
\frac{\int_{\Omega}\left|\nabla u_{\varepsilon}\right|^{p} d x}{\left(\int_{\Omega} h(x)\left|u_{\varepsilon}\right|^{p^{*}}\right)^{p / p^{*}}}=S+O\left(\varepsilon^{(N-p) / p}\right)
$$

where $U(x)=\left(1+|x|^{p /(p-1)}\right)^{-(N-p) / p}$.

Set $u_{0}=\sqrt[p]{\alpha} u_{\varepsilon}, v_{0}=\sqrt[p]{\beta} u_{\varepsilon}$, and $z_{0}=\left(u_{0}, v_{0}\right) \in W$. Then, from (4.8), (4.23), and $|h|_{\infty}=1$, we conclude that

$$
\begin{aligned}
\sup _{t \geq 0}\left(t z_{0}\right) & \leq \frac{1}{N}\left(\frac{(\alpha+\beta) \int_{\Omega}\left|\nabla u_{\varepsilon}\right|^{p} d x}{\left(\alpha^{\alpha / p} \beta^{\beta / p} \int_{\Omega} h(x)\left|u_{\varepsilon}\right|^{p^{*}}\right)^{p / p^{*}}}\right)^{N / p} \\
& \leq \frac{1}{N}\left(\left(\frac{\alpha}{\beta}\right)^{\beta /(\alpha+\beta)}+\left(\frac{\beta}{\alpha}\right)^{\alpha /(\alpha+\beta)}\right)^{N / p}\left(S+O\left(\varepsilon^{(N-p) / p}\right)\right)^{N / p} \\
& =\frac{1}{N}\left(\left(\frac{\alpha}{\beta}\right)^{\alpha /(\alpha+\beta)}+\left(\frac{\beta}{\alpha}\right)^{\alpha /(\alpha+\beta)}\right)^{N / p}\left(S^{N / p}+O\left(\varepsilon^{(N-p) / p}\right)\right) \\
& =\frac{1}{N} S_{\alpha, \beta}^{N / p}+O\left(\varepsilon^{(N-p) / p}\right),
\end{aligned}
$$

where the following fact has been used:

$$
\sup _{t \geq 0}\left(\frac{t^{p}}{p} A-\frac{t^{\alpha+\beta}}{\alpha+\beta} B\right)=\frac{1}{N} A\left(\frac{A}{B}\right)^{(N-p) / p}=\frac{1}{N}\left(\frac{A}{B^{p / p^{*}}}\right)^{N / p}, \quad A, B>0 .
$$

Using the definitions of $I_{\lambda, \mu}, z_{0}$ and by $(A 3)$ and $(A 4)$, we get

$$
I_{\lambda, \mu}\left(t z_{0}\right) \leq \frac{t^{p}}{p}\left\|z_{0}\right\|^{p}=\frac{\alpha+\beta}{p} t^{p}\left|\nabla u_{\varepsilon}\right|_{p}^{p} \quad \forall t \geq 0, \lambda, \mu>0
$$

Combining this with (4.22), let $\varepsilon \in(0,1)$, then there exists $t_{0} \in(0,1)$ independent of $\varepsilon$ such that

$$
\sup _{0 \leq t \leq t_{0}} I_{\lambda, \mu}\left(t z_{0}\right)<c_{\infty}, \quad \forall \lambda, \mu>0, \forall \varepsilon \in(0,1)
$$

Using the definitions of $I_{\lambda, \mu}, z_{0}$ and by $\alpha, \beta>1,(4.24)$ and (A3), we have 


$$
\begin{aligned}
\sup _{t \geq t_{0}} I_{\lambda, \mu}\left(t z_{0}\right) & =\sup _{t \geq t_{0}}\left(Q\left(t z_{0}\right)-\frac{t^{q}}{q} K_{\lambda, \mu}\left(t z_{0}\right)\right) \\
& \leq \frac{1}{N} S_{\alpha, \beta}^{N / p}+O\left(\varepsilon^{(N-p) / p}\right)-\frac{t_{0}^{q}}{q}\left(a_{0} \alpha^{q / p} \lambda+b_{0} \beta^{q / p} \mu\right) \int_{B\left(0, \rho_{0}\right)}\left|u_{\varepsilon}\right|^{q} d x \\
& \leq \frac{1}{N} S_{\alpha, \beta}^{N / p}+O\left(\varepsilon^{(N-p) / p}\right)-\frac{t_{0}^{q}}{q} \gamma_{0}(\lambda+\mu) \int_{B\left(0, \rho_{0}\right)}\left|u_{\varepsilon}\right|^{q} d x
\end{aligned}
$$

where $\gamma_{0}=\min \left\{a_{0} \alpha^{q / p}, b_{0} \beta^{q / p}\right\}$.

Let $0<\varepsilon \leq \rho_{0}^{p /(p-1)}$; we have

$$
\begin{aligned}
\int_{B\left(0, \rho_{0}\right)}\left|u_{\varepsilon}\right|^{q} d x & =\int_{B\left(0, \rho_{0}\right)} \frac{\varepsilon^{q(N-p) / p^{2}}}{\left(\varepsilon+|x|^{p /(p-1)}\right)^{((N-p) / p) q}} d x \\
& \geq \int_{B\left(0, \rho_{0}\right)} \frac{\varepsilon^{q(N-p) / p^{2}}}{\left(2 \rho_{0}^{p /(p-1)}\right)^{((N-p) / p) q}} d x \\
& =C_{1}\left(N, p, q, \rho_{0}\right) \varepsilon^{q(N-p) / p^{2}} .
\end{aligned}
$$

Combining with (4.28) and (4.29), for all $\varepsilon \in\left(0, \rho_{0}^{p /(p-1)}\right)$, we get

$$
\sup _{t \geq t_{0}} I_{\lambda, \mu}\left(t z_{0}\right) \leq \frac{1}{N} S_{\alpha, \beta}^{N / p}+O\left(\varepsilon^{(N-p) / p}\right)-\frac{t_{0}^{q}}{q} C_{1} \gamma_{0}(\lambda+\mu) \varepsilon^{q(N-p) / p^{2}}
$$

Hence, for any $\lambda, \mu>0$, we can choose small positive constant $\varepsilon_{\lambda, \mu}<\min \left\{1, \rho_{0}^{p /(p-1)}\right\}$ such that

$$
O\left(\varepsilon_{\lambda, \mu}^{(N-p) / p}\right)-\frac{t_{0}^{q}}{q} C_{1} \gamma_{0}(\lambda+\mu) \varepsilon_{\lambda, \mu}^{q(N-p) / p^{2}}<0
$$

Now, we fix $\varepsilon_{\lambda, \mu}$ and let $z_{\lambda, \mu}=\left(\sqrt[p]{\alpha} u_{\varepsilon_{1, \mu}}, \sqrt[p]{\beta} u_{\varepsilon_{\lambda, \mu}}\right)$. From (4.27), (4.30), (4.31), we can deduce that, for any $\lambda, \mu>0$, there exists a nonnegative function $z_{\lambda, \mu} \in W \backslash\{0\}$ such that

$$
\sup _{t \geq 0} I_{\lambda, \mu}\left(t z_{\lambda, \mu}\right)<c_{\infty}
$$

Finally, we prove that $\theta_{\lambda, \mu}^{-}<c_{\infty}$ for all $0<\lambda^{p /(p-q)}+\mu^{p /(p-q)}<\Lambda_{1}$. Recall that $z_{\lambda, \mu}=\left(u_{\lambda, \mu}, v_{\lambda, \mu}\right)=$ $\left(\sqrt[p]{\alpha} u_{\varepsilon_{\lambda, \mu},}, \sqrt[p]{\beta} u_{\varepsilon_{\lambda, \mu}}\right)$. By $(A 3),(A 4)$, and the definition of $u_{\varepsilon_{\lambda, \mu}}$, we have

$$
J\left(z_{\lambda, \mu}\right)>0, \quad K_{\lambda, \mu}\left(z_{\lambda, \mu}\right)>0 .
$$


Combining this with Lemma 2.6 (ii), from the definition of $\theta_{\lambda, \mu}^{-}$and (4.32), for all $0<\lambda^{p /(p-q)}+$ $\mu^{p /(p-q)}<\Lambda_{1}$, we obtain that there exists $t_{\lambda, \mu}>0$ such that $t_{\lambda, \mu} z_{\lambda, \mu} \in N_{\lambda, \mu}^{-}$and

$$
\theta_{\lambda, \mu}^{-} \leq I_{\lambda, \mu}\left(t_{\lambda, \mu} z_{\lambda, \mu}\right) \leq \sup _{t \geq 0} I_{\lambda, \mu}\left(t z_{\lambda, \mu}\right)<c_{\infty}
$$

This completes the proof.

Theorem 4.4. Assume that $(A 1)-(A 4)$ hold. If $0<\lambda^{p /(p-q)}+\mu^{p /(p-q)}<(q / p)^{p /(p-q)} \Lambda_{1}$, then $I_{\lambda, \mu}$ satisfies the $(P S)_{\theta_{\lambda, \mu}^{-}}$condition. Moreover, $I_{\lambda, \mu}$ has a minimizer $z_{\lambda, \mu}^{2}$ in $N_{\lambda, \mu}^{-}$and satisfies the following:

(i) $I_{\lambda, \mu}\left(z_{\lambda, \mu}^{2}\right)=\theta_{\lambda, \mu^{\prime}}^{-}$

(ii) $z_{\lambda, \mu}^{2}$ is a positive solution of System $\left(S_{\lambda f, \mu g, h}\right)$, where $\Lambda_{1}$ is the same as in (1.6).

Proof. If $0<\lambda^{p /(p-q)}+\mu^{p /(p-q)}<(q / p)^{p /(p-q)} \Lambda_{1}$, then by Theorem 2.5 (ii), Proposition 3.2 (ii), and Lemma 4.3, there exists a (PS) ${\theta_{\lambda, \mu}^{-}}^{-}$-sequence $\left\{z_{n}\right\} \subset \mathcal{N}_{\lambda, \mu}^{-}$in $W$ for $I_{\lambda, \mu}$ with $\theta_{\lambda, \mu}^{-} \in\left(0, c_{\infty}\right)$. From Lemma 4.2, there exist a subsequence still denoted by $\left\{z_{n}\right\}$ and a nontrivial solution $z_{\lambda, \mu}^{2}=\left(u_{\lambda, \mu^{\prime}}^{2} v_{\lambda, \mu}^{2}\right) \in W$ of System $\left(S_{\lambda f, \mu g, h}\right)$ such that $z_{n} \rightarrow z_{\lambda, \mu}^{2}$ weakly in $W$. Now we prove that $z_{n} \rightarrow z_{\lambda, \mu}^{2}$ strongly in $W$ and $I_{\lambda, \mu}\left(z_{\lambda, \mu}^{2}\right)=\theta_{\lambda, \mu}^{-}$. By (3.4), if $z \in \mathcal{N}_{\lambda, \mu}$, then

$$
I_{\lambda, \mu}(z)=\frac{p^{*}-p}{p^{*} p}\|z\|^{p}-\frac{p^{*}-q}{p^{*} q} K_{\lambda, \mu}(z)
$$

First, we prove that $z_{\lambda, \mu}^{2} \in \mathcal{N}_{\lambda, \mu}^{-}$. On the contrary, if $z_{\lambda, \mu}^{2} \in \mathcal{N}_{\lambda, \mu^{\prime}}^{+}$then by $\mathcal{N}_{\lambda, \mu}^{-}$closed in $W$, we have $\left\|z_{\lambda, \mu}^{2}\right\|<\lim _{n \rightarrow \infty}\left\|z_{n}\right\|$. By Lemma $2.3(i)$ and (A4), we obtain that

$$
K_{\lambda, \mu}\left(z_{\lambda, \mu}^{2}\right)>0, \quad J\left(z_{\lambda, \mu}^{2}\right)>0
$$

By Lemma 2.7, there exists a unique $t^{-}$such that $t^{-} z_{\lambda, \mu}^{2} \in \mathcal{N}_{\lambda, \mu}^{-}$. Since $z_{n} \in \mathcal{N}_{\lambda, \mu^{\prime}}^{-} I_{\lambda, \mu}\left(z_{n}\right) \geq$ $I_{\lambda, \mu}\left(t z_{n}\right)$ for all $t \geq 0$ and by (4.35), we have

$$
\theta_{\lambda, \mu}^{-} \leq I_{\lambda, \mu}\left(t^{-} z_{\lambda, \mu}^{2}\right)<\lim _{n \rightarrow \infty} I_{\lambda, \mu}\left(t^{-} z_{n}\right) \leq \lim _{n \rightarrow \infty} I_{\lambda, \mu}\left(z_{n}\right)=\theta_{\lambda, \mu^{\prime}}^{-}
$$

and this is a contradiction.

In order to prove that $I_{\lambda, \mu}\left(z_{\lambda, \mu}^{2}\right)=\theta_{\lambda, \mu}^{-}$it suffices to recall that $z_{n}, z_{\lambda, \mu}^{2} \in \mathcal{N}_{\lambda, \mu}^{-}$for all $n$, by (4.35). and apply Fatou's lemma to get

$$
\begin{aligned}
\theta_{\lambda, \mu}^{-} & \leq I_{\lambda, \mu}\left(z_{\lambda, \mu}^{2}\right)=\frac{p^{*}-p}{p^{*} p}\left\|z_{\lambda, \mu}^{2}\right\|^{p}-\frac{p^{*}-q}{p^{*} q} K_{\lambda, \mu}\left(z_{\lambda, \mu}^{2}\right) \\
& \leq \liminf _{n \rightarrow \infty}\left(\frac{p^{*}-p}{p^{*} p}\left\|z_{n}\right\|^{p}-\frac{p^{*}-q}{p^{*} q} K_{\lambda, \mu}\left(z_{n}\right)\right) \\
& \leq \liminf _{n \rightarrow \infty} I_{\lambda, \mu}\left(z_{n}\right)=\theta_{\lambda, \mu}^{-} .
\end{aligned}
$$


This implies that $I_{\lambda, \mu}\left(z_{\lambda, \mu}^{2}\right)=\theta_{\lambda, \mu}^{-}$and $\lim _{n \rightarrow \infty}\left\|z_{n}\right\|^{p}=\left\|z_{\lambda, \mu}^{2}\right\|^{p}$. Let $\widetilde{z}_{n}=z_{n}-z_{\lambda, \mu^{\prime}}^{2}$ then by Brézis and Lieb lemma [26] implies

$$
\left\|\tilde{z}_{n}\right\|^{p}=\left\|z_{n}\right\|^{p}-\left\|z_{\lambda, \mu}^{2}\right\|^{p}
$$

Therefore, $z_{n} \rightarrow z_{\lambda, \mu}^{2}$ strongly in $W$.

Since $I_{\lambda, \mu}\left(z_{\lambda, \mu}^{2}\right)=I_{\lambda, \mu}\left(\left|z_{\lambda, \mu}^{2}\right|\right)$ and $\left|z_{\lambda, \mu}^{2}\right| \in \mathcal{N}_{\lambda, \mu}^{-}$by Lemmas 2.2, and 2.3 (iii), we may assume that $z_{\lambda, \mu}^{2}$ is a nontrivial nonnegative solution of System $\left(S_{\lambda f, \mu g, h}\right)$. Finally, by using the same arguments as in the proof of Theorem 3.3, for all $0<\lambda^{p /(p-q)}+\mu^{p /(p-q)}<(q / p)^{p /(p-q)} \Lambda_{1}$, we have that $z_{\lambda, \mu}^{2}$ is a positive solution of System $\left(S_{\lambda f, \mu g, h}\right)$.

Proof of Theorem 1.3. By Theorems 3.3, and 4.4, we obtain that for all $\lambda, \mu>0$ and $0<\lambda^{p /(p-q)}+$ $\mu^{p /(p-q)}<(q / p)^{p /(p-q)} \Lambda_{1},\left(S_{\lambda f, \mu g, h}\right)$ has two positive solutions $z_{\lambda, \mu^{\prime}}^{1} z_{\lambda, \mu}^{2}$ with $z_{\lambda, \mu}^{1} \in \mathcal{N}_{\lambda, \mu^{\prime}}^{+} z_{\lambda, \mu}^{2} \in$ $\mathcal{N}_{\lambda, \mu}^{-}$. Since $\mathcal{N}_{\lambda, \mu}^{+} \cap \mathcal{N}_{\lambda, \mu}^{-}=\emptyset$, this implies that $z_{\lambda, \mu}^{1}$ and $z_{\lambda, \mu}^{2}$ are distinct. This completes the proof of Theorem 1.3.

\section{References}

[1] A. Ambrosetti, H. Brezis, and G. Cerami, "Combined effects of concave and convex nonlinearities in some elliptic problems," Journal of Functional Analysis, vol. 122, no. 2, pp. 519-543, 1994.

[2] D. G. de Figueiredo, J.-P. Gossez, and P. Ubilla, "Local "superlinearity" and "sublinearity" for the -Laplacian," Journal of Functional Analysis, vol. 257, no. 3, pp. 721-752, 2009.

[3] J. G. Azorero and I. P. Peral, "Some results about the existence of a second positive solution in a quasilinear critical problem," Indiana University Mathematics Journal, vol. 43, no. 3, pp. 941-957, 1994.

[4] J. P. G. Azorero, I. Peral, and J. J. Manfredi, "Sobolev versus Hölder local minimizers and global multiplicity for some quasilinear elliptic equations," Communications in Contemporary Mathematics, vol. 2, no. 3, pp. 385-404, 2000.

[5] A. El Hamidi, "Multiple solutions with changing sign energy to a nonlinear elliptic equation," Communications on Pure and Applied Analysis, vol. 3, no. 2, pp. 253-265, 2004.

[6] N. Hirano, C. Saccon, and N. Shioji, "Existence of multiple positive solutions for singular elliptic problems with concave and convex nonlinearities," Advances in Differential Equations, vol. 9, no. 1-2, pp. 197-220, 2004.

[7] T.-S. Hsu, "Multiplicity results for $P$-Laplacian with critical nonlinearity of concave-convex type and sign-changing weight functions," Abstract and Applied Analysis, Article ID 652109, 24 pages, 2009.

[8] T.-F. Wu, "On semilinear elliptic equations involving concave-convex nonlinearities and signchanging weight function," Journal of Mathematical Analysis and Applications, vol. 318, no. 1, pp. 253$270,2006$.

[9] K. Adriouch and A. El Hamidi, "The Nehari manifold for systems of nonlinear elliptic equations," Nonlinear Analysis. Theory, Methods E Applications, vol. 64, no. 10, pp. 2149-2167, 2006.

[10] A. Ahammou, "Positive radial solutions of nonlinear elliptic systems," New York Journal of Mathematics, vol. 7, pp. 267-280, 2001.

[11] C. O. Alves, D. C. D. M. Filho, and M. A. S. Souto, “On systems of elliptic equations involving subcritical or critical Sobolev exponents," Nonlinear Analysis. Theory, Methods \& Applications, vol. 42, no. 5, pp. 771-787, 2000.

[12] Y. Bozhkov and E. Mitidieri, "Existence of multiple solutions for quasilinear systems via fibering method," Journal of Differential Equations, vol. 190, no. 1, pp. 239-267, 2003.

[13] Ph. Clément, D. G. de Figueiredo, and E. Mitidieri, "Positive solutions of semilinear elliptic systems," Communications in Partial Differential Equations, vol. 17, no. 5-6, pp. 923-940, 1992.

[14] D. G. de Figueiredo and P. L. Felmer, "On superquadratic elliptic systems," Transactions of the American Mathematical Society, vol. 343, no. 1, pp. 99-116, 1994. 
[15] A. El Hamidi, "Existence results to elliptic systems with nonstandard growth conditions," Journal of Mathematical Analysis and Applications, vol. 300, no. 1, pp. 30-42, 2004.

[16] T.-S. Hsu, "Multiple positive solutions for a critical quasilinear elliptic system with concave-convex nonlinearities," Nonlinear Analysis. Theory, Methods \& Applications, vol. 71, no. 7-8, pp. 2688-2698, 2009.

[17] T.-S. Hsu and H.-L. Lin, "Multiple positive solutions for a critical elliptic system with concave-convex nonlinearities," Proceedings of the Royal Society of Edinburgh. Section A. Mathematics, vol. 139, no. 6, pp. 1163-1177, 2009.

[18] M. Squassina, "An eigenvalue problem for elliptic systems," New York Journal of Mathematics, vol. 6, pp. 95-106, 2000.

[19] J. Vélin, "Existence results for some nonlinear elliptic system with lack of compactness," Nonlinear Analysis. Theory, Methods E Applications, vol. 52, no. 3, pp. 1017-1034, 2003.

[20] T.-F. Wu, "The Nehari manifold for a semilinear elliptic system involving sign-changing weight functions," Nonlinear Analysis. Theory, Methods \& Applications, vol. 68, no. 6, pp. 1733-1745, 2008.

[21] Z. Nehari, "On a class of nonlinear second-order differential equations," Transactions of the American Mathematical Society, vol. 95, pp. 101-123, 1960.

[22] M. Willem, Minimax Theorems, Birkhäuser Boston, Boston, Mass, USA, 1996.

[23] G. Tarantello, "On nonhomogeneous elliptic equations involving critical Sobolev exponent," Annales de l'Institut Henri Poincaré. Analyse Non Linéaire, vol. 9, no. 3, pp. 281-304, 1992.

[24] K. J. Brown and Y. Zhang, "The Nehari manifold for a semilinear elliptic equation with a signchanging weight function," Journal of Differential Equations, vol. 193, no. 2, pp. 481-499, 2003.

[25] K. J. Brown and T.-F. Wu, "A semilinear elliptic system involving nonlinear boundary condition and sign-changing weight function," Journal of Mathematical Analysis and Applications, vol. 337, no. 2, pp. 1326-1336, 2008.

[26] H. Brézis and E. Lieb, "A relation between pointwise convergence of functions and convergence of functionals," Proceedings of the American Mathematical Society, vol. 88, no. 3, pp. 486-490, 1983.

[27] N.S. Trudinger, "On Harnack type inequalities and their application to quasilinear elliptic equations," Communications on Pure and Applied Mathematics, vol. 20, pp. 721-747, 1967. 


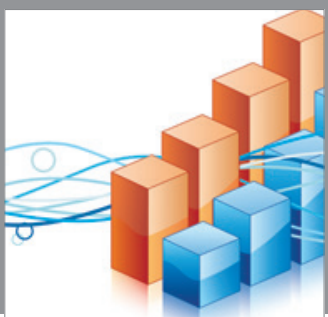

Advances in

Operations Research

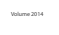

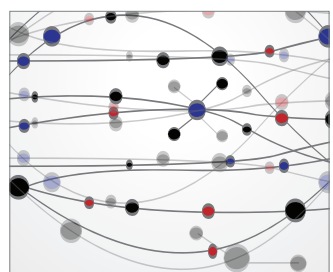

\section{The Scientific} World Journal
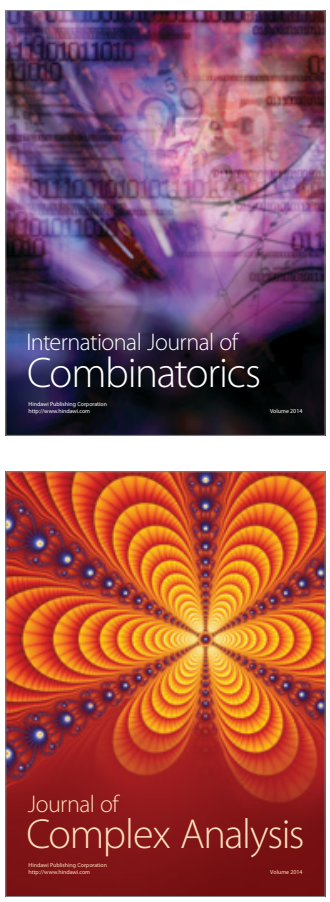

International Journal of

Mathematics and

Mathematical

Sciences
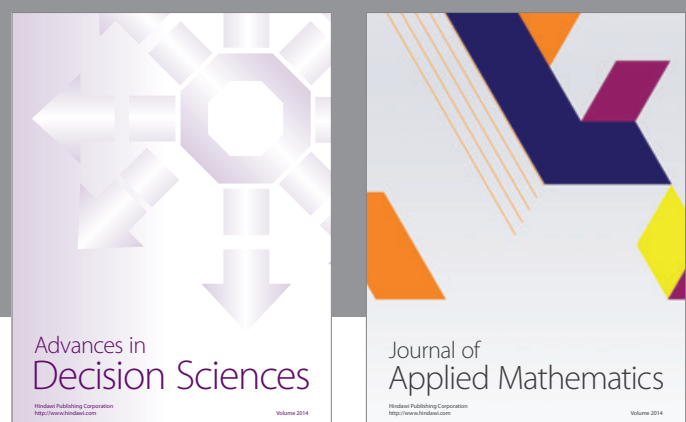

Journal of

Applied Mathematics
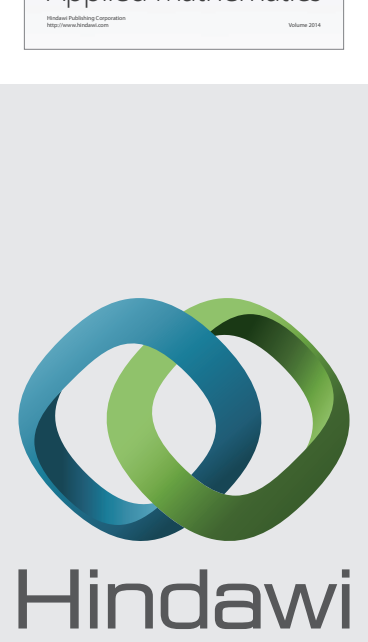

Submit your manuscripts at http://www.hindawi.com
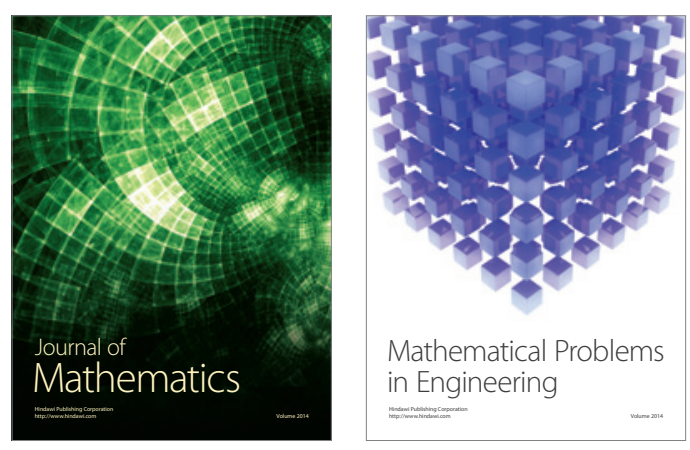

Mathematical Problems in Engineering
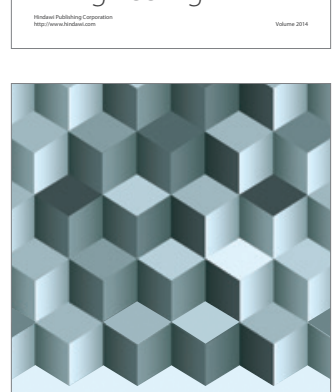

Journal of

Function Spaces
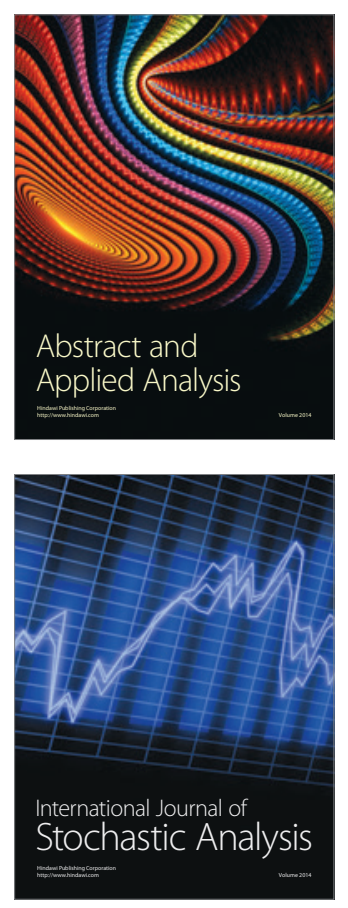

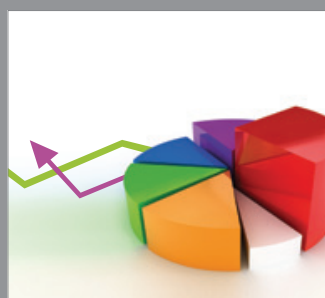

ournal of

Probability and Statistics

Promensencen
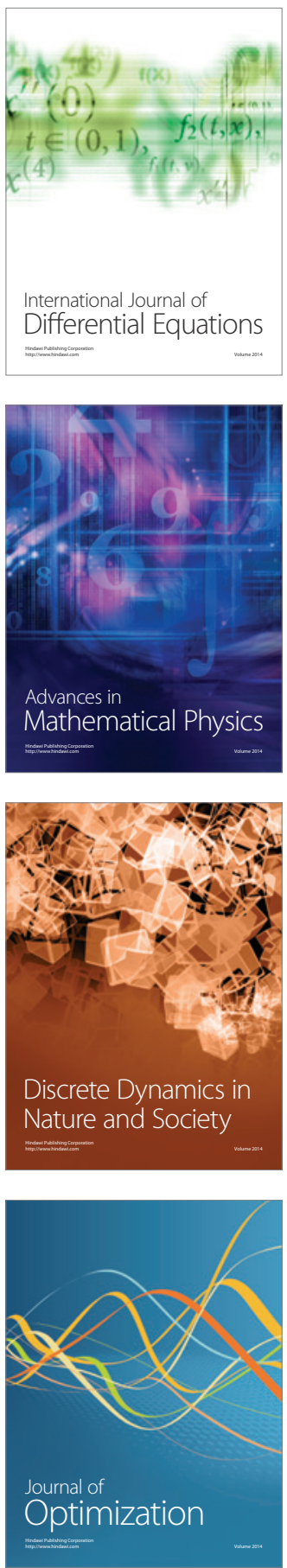